\title{
A FACTORIZATION THEOREM FOR UNFOLDINGS OF ANALYTIC FUNCTIONS
}

\author{
TATSUO SUWA
}

(Communicated by Irwin Kra)

\begin{abstract}
Let $\tilde{f}$ and $g$ be holomorphic function germs at $\mathbf{0}$ in $\mathbf{C}^{n} \times \mathbf{C}^{l}=$ $\{(x, s)\}$. If $d_{x} g \wedge d_{x} \tilde{f}=0$ and if $f(x)=\tilde{f}(x, 0)$ is not a power or a unit, then there exists a germ $\lambda$ at 0 in $\mathbf{C} \times \mathbf{C}^{l}$ such that $g(x, s)=\lambda(\tilde{f}(x, s), s)$. The result has the implication that the notion of an RL-morphism in the unfolding theory of foliation germs generalizes that of a right-left morphism in the function germ case.
\end{abstract}

The notion of an RL-morphism in the unfolding theory of foliation singularities was introduced in [5] to describe the determinacy results and in [6] the versality theorem for these morphisms is proved. This note, which should be considered as an appendix to [5 or 6], contains a factorization theorem implying that an RLmorphism is a generalization of a right-left morphism in the unfolding theory of function germs. It depends on the Mattei-Moussu factorization theorem [1] and is a generalization of a result of Moussu [2].

A codim 1 foliation germ at 0 in $\mathbf{C}^{n}$ is a module $F=(\omega)$ over the ring of holomorphic function germs generated by a germ of an integrable 1-form $\omega$ (see $\S 2$ ). An unfolding of $F$ with parameter space $\mathbf{C}^{m}=\{t\}$ is a codim 1 foliation germ $\mathscr{F}=(\tilde{\omega})$ at 0 in $\mathbf{C}^{n} \times \mathbf{C}^{m}$ with a generator $\tilde{\omega}$ whose restriction to $\mathbf{C}^{n} \times\{0\}$ is $\omega$. We let $F_{t}$ be the foliation germ generated by the restriction $\omega_{t}$ of $\tilde{\omega}$ to $\mathbf{C}^{n} \times\{t\}$. Let $\mathscr{F}^{\prime}$ be another unfolding of $F$ with parameter space $\mathbf{C}^{l}=\{s\}$. A morphism from $\mathscr{F}^{\prime}$ to $\mathscr{F}$ is a holomorphic map germ $\Phi:\left(\mathbf{C}^{n} \times \mathbf{C}^{l}, 0\right) \rightarrow\left(\mathbf{C}^{n} \times \mathbf{C}^{m}, 0\right)$ such that (a) $\Phi(x, s)=(\phi(s, x), \psi(s))$ for some holomorphic map germs $\phi:\left(\mathbf{C}^{n} \times \mathbf{C}^{l}, 0\right) \rightarrow\left(\mathbf{C}^{n}, 0\right)$ and $\psi:\left(\mathbf{C}^{l}, 0\right) \rightarrow\left(\mathbf{C}^{m}, 0\right)$, (b) $\phi(x, 0)=x$ and (c) the pull back $\Phi^{*} \tilde{\omega}$ of $\tilde{\omega}$ by $\Phi$ generates $\mathscr{F}^{\prime}$. Thus, if we set $\phi_{s}(x)=\phi(x, s)$, we may think of $\left(\phi_{s}\right)$ as a family of local coordinate changes of $\left(\mathbf{C}^{n}, 0\right)$. For an RL-morphism, in place of $(\mathbf{c})$, we only require that $\phi_{s}^{*} \omega_{\psi(s)}$ generates $F_{s}^{\prime}$ for each $s$ (see (2.1) Definition). Our previous result shows that if $F$ has a generator of the form $d f$ for some holomorphic function germ $f$ (strong first integral for $F$ ), then every unfolding of $F$ admits a generator of the form $d \tilde{f}$ with $\tilde{f}$ an unfolding of $f$. In the unfolding theory of function germs, there are notions of a right morphism and a right-left morphism. The former involves coordinate changes in the source space $\left(\mathbf{C}^{n}, 0\right)$, whereas the latter involves coordinate changes in the target space $\mathbf{C}$ as well. It is not difficult to see that our morphism generalizes a right morphism in the sense that when $F$ admits a strong first integral $f$, then it becomes a (strict) right morphism in the unfolding theory of $f$. For a foliation without first integrals, it may not seem relevant to talk about

Received by the editors May 5, 1987 and, in revised form, July 20, 1987.

1980 Mathematics Subject Classification (1985 Revision). Primary 32A10, 32G11; Secondary $58 \mathrm{C} 27,58 \mathrm{~F} 14$. 
right-left morphisms. However, as stated above, our factorization theorem shows that an RL-morphism is a natural generalization of a right-left morphism, since when $F=(d f)$, an RL-morphism is exactly a right-left morphism in the unfolding theory of $f$. We also note that RL-morphisms are closely related to integrating factors of the foliation ((2.2) Remark 2).

1. The factorization theorem. We denote by $\mathscr{O}_{n}$ the ring of germs of holomorphic functions at the origin 0 in $\mathbf{C}^{n}=\left\{\left(x_{1}, \ldots, x_{n}\right)\right\}$. A germ $f$ in $\mathscr{O}_{n}$ is said to be a power if $f=f_{0}^{m}$ for some positive integer $m$ and a nonunit $f_{0}$ in $\mathscr{O}_{n}$. If we denote the critical set of $f$ by $C(f)$, then codim $C(f) \geq 2$ implies that $f$ is not a power. We quote the following factorization theorem of Mattei and Moussu.

(1.1) THEOREM [1]. Let $f$ be a germ in $\mathscr{O}_{n}$ which is not a power or a unit. If $g$ is a germ in $\mathscr{O}_{n}$ with $d g \wedge d f=0$, then there exists a germ $\lambda$ in $\mathscr{O}_{1}$ such that $g=\lambda \circ f$.

The theorem is proved using the reduction theory of singularities of holomorphic 1-forms due to Seidenberg and Van den Essen. The proof is rather simple if we assume codim $C(f) \geq 2$ (see Moussu-Tougeron [3]). If $\tilde{f}$ is a germ in $\mathscr{O}_{n+l}$, we may think of $\tilde{f}$ as an unfolding of $f(x)=\tilde{f}(x, 0)$ with parameter space $C^{l}=$ $\left\{\left(s_{1}, \ldots, s_{l}\right)\right\}$. We denote by $d_{x}$ the exterior derivation with respect to $x ; d_{x} \tilde{f}=$ $\sum_{i=1}^{n} \partial \tilde{f} / \partial x_{i}(s, x) d x_{i}$.

(1.2) THEOREM. Let $\tilde{f}$ be a germ in $\mathscr{O}_{n+l}$ such that $f(x)=\tilde{f}(x, 0)$ is not a power or a unit in $\mathscr{O}_{n}$. If $g$ is a germ in $\mathscr{O}_{n+l}$ with $d_{x} g \wedge d_{x} \tilde{f}=0$, then there exists a germ $\lambda$ in $\mathscr{O}_{1+l}$ such that $g(x, s)=\lambda(\tilde{f}(x, s), s)$.

ProOF. First we show the existence of $\lambda$ as a formal power series in $s$. Thus we express $\lambda$ as

$$
\lambda(y, s)=\sum_{|\nu| \geq 0} \lambda^{(\nu)}(y) s^{\nu}, \quad \lambda^{(\nu)} \in \mathscr{O}_{1}
$$

where $\nu$ denotes an $l$-tuple $\left(\nu_{1}, \ldots, \nu_{l}\right)$ of nonnegative integers, $|\nu|=\nu_{1}+\cdots+\nu_{l}$ and $s^{\nu}=s_{1}^{\nu_{1}} \cdots s_{l}^{\nu_{l}}$. In general, if $\sigma=\sum_{|\nu| \geq 0} \sigma^{(\nu)} s^{\nu}$ is a series in $s$ with $\sigma^{(\nu)} \in \mathscr{O}_{n}^{r}$ for some $r$, we set

$$
[\sigma]_{p}=\sum_{|\nu|=p} \sigma^{(\nu)} s^{\nu} \quad \text { and } \quad \sigma^{\mid p}=\sum_{|\nu|=0}^{p} \sigma^{(\nu)} s^{\nu}
$$

for a nonnegative integer $p$.

We look for $\lambda$ satisfying the congruence

$$
g(x, s) \equiv_{p} \lambda^{\mid p}(\tilde{f}(x, s), s)
$$

for $p \geq 0$, where $\equiv_{p}$ denotes equality $\bmod s^{\nu},|\nu|=p+1$. First, $(1.3)_{0}$ is equivalent to

$$
g(x, 0)=\lambda^{(0)}(f(x))
$$

From the condition of the theorem, we have $d(g(x, 0)) \wedge d f=0$. Hence by (1.1), there exists a germ $\lambda^{(0)}$ in $\mathscr{O}_{1}$ satisfying the above. Now we suppose that we have $\lambda^{\mid p}$ satisfying $(1.3)_{p}$ and look for $[\lambda]_{p+1}$. The congruence $(1.3)_{p+1}$ reads

$$
g(x, s) \equiv_{p+1} \sum_{|\nu|=p+1} \lambda^{(\nu)}(f(x)) s^{\nu}+\lambda^{\mid p}(\tilde{f}(x, s), s) .
$$


Hence, for our purpose, it suffices to show that

$$
d_{x}\left[g(x, s)-\lambda^{\mid p}(\tilde{f}(x, s), s)\right]_{p+1} \wedge d f=0 .
$$

By $(1.3)_{p}$, we have (1.4) if we show that

$$
d_{x}\left(g(x, s)-\lambda^{\mid p}(\tilde{f}(x, s), s)\right) \wedge d_{x} \tilde{f} \equiv_{p+1} 0 .
$$

But this follows from the condition of the theorem and

$$
d_{x} \lambda^{\mid p}(\tilde{f}(x, s), s)=\frac{\partial \lambda^{\mid p}}{\partial y}(\tilde{f}(x, s), s) d_{x} \tilde{f} .
$$

Thus we have a formal power series

$$
\lambda(y, s)=\sum_{|\nu| \geq 0} \lambda^{(\nu)}(y) s^{\nu}, \quad \lambda^{(\nu)} \in \mathscr{O}_{1},
$$

in $s$ such that $g(x, s)=\lambda(\tilde{f}(x, s), s)$ as power series in $(x, s)$. Since $\tilde{f}$ and $g$ are both convergent, $\lambda$ must be also convergent.

(1.5) REMARKS. 1. The germ $\lambda$ is determined uniquely by $g$ (and $\tilde{f}$ ). If we assume that $g(x, 0)=f(x)$, then $\lambda(y, 0)=y$.

2. The above theorem generalizes Corollaire 1 in $[2$, Chapter II, 1] in the case $X=H$.

2. Some types of morphisms in the unfolding theory of foliation germs. We denote by $\Omega_{n}$ the $\mathscr{O}_{n}$-modules of germs of holomorphic 1 -forms at 0 in $\mathbf{C}^{n}$. We recall (cf. $\left.[\mathbf{4}, \mathbf{5}]\right)$ that a codim 1 foliation germ at 0 in $\mathbf{C}^{n}$ is a rank 1 free sub- $\mathscr{O}_{n}$-module $F=(\omega)$ of $\Omega_{n}$ with a generator satisfying the integrability condition $d \omega \wedge \omega=0$. The singular set $S(F)$ of $F$ is defined to be the singular set $\{x \mid \omega(x)=0\}$ of $\omega$. We always assume that codim $S(F) \geq 2$. An unfolding of $F=(\omega)$ is a codim 1 foliation germ $\mathscr{F}=(\tilde{\omega})$ at 0 in $\mathbf{C}^{n} \times \mathbf{C}^{m}=\{(x, t)\}$, for some $m$, with a generator $\tilde{\omega}$ satisfying $\iota^{*} \tilde{\omega}=\omega$, where $\iota$ denotes the embedding of $\mathbf{C}^{n}$ into $\mathbf{C}^{n} \times \mathbf{C}^{m}$ given by $\iota(x)=(x, 0)$. We call $\mathbf{C}^{m}$ the parameter space of $\mathscr{F}$. We recall the following definition $[5,(2.1), 6,(1.1)]$.

(2.1) DEFINITION. Let $\mathscr{F}$ and $\mathscr{F}^{\prime}$ be two unfoldings of $F$ with parameter spaces $\mathbf{C}^{m}$ and $\mathbf{C}^{l}=\left\{\left(s_{1}, \ldots, s_{l}\right)\right\}$, respectively.

(I) An RL-morphism from $\mathscr{F}^{\prime}$ to $\mathscr{F}$ is a pair $(\Phi, \psi)$ satisfying the following conditions.

(a) $\Phi$ and $\psi$ are holomorphic map germs making the diagram

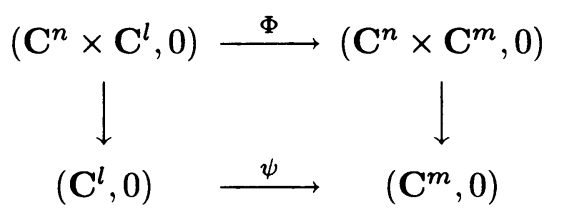

commutative, where the vertical maps are the projections.

(b) $\Phi(x, 0)=(x, 0)$.

(c) For any generator $\tilde{\omega}$ of $\mathscr{F}$, there is a germ $\alpha=\left(\alpha_{1}, \ldots, \alpha_{l}\right)$ in $\mathscr{O}_{n+l}^{l}$ such that the germ

$$
\Phi^{*} \tilde{\omega}+\sum_{k=1}^{l} \alpha_{k} d s_{k}
$$

generates $\mathscr{F}^{\prime}$. 
(II) A morphism from $\mathscr{F}^{\prime}$ to $\mathscr{F}$ is an RL-morphism such that for any generator $\tilde{\omega}$ of $\mathscr{F}$, we may choose $\alpha=0$ in (c).

(2.2) REMARKS. 1. In both cases, we may replace "any" by "some".

2. From the integrability condition we see that, for $\alpha$ in (c), each $\alpha_{k}(x, 0)$ is an integrating factor of $\omega=\iota^{*} \tilde{\omega}$, i.e., $\alpha_{k}(x, 0) d \omega=d\left(\alpha_{k}(x, 0)\right) \wedge \omega$.

3 . We have a "versality theorem" for each type of morphisms $[4,6]$.

If a germ $\tilde{f}$ in $\mathscr{O}_{n+m}$ is an unfolding of $f$, i.e., if $\iota^{*} \tilde{f}=f$, then $\mathscr{F}=(d \tilde{f})$ is an unfolding of $F=(d f)$ with parameter space $\mathbf{C}^{m}$ and conversely, any unfolding of $F=(d f)$ has a generator of the form $d \tilde{f}$ with $\tilde{f}$ an unfolding of $f[\mathbf{4}$, p. 47]. We recall the following definition (cf. [7, Definition 3.2]).

(2.3) DEFINITION. Let $\tilde{f}$ and $g$ be two unfoldings of $f$ with parameter spaces $\mathbf{C}^{m}$ and $\mathbf{C}^{l}=\left\{\left(s_{1}, \ldots, s_{l}\right)\right\}$, respectively.

(I) A right-left morphism from $g$ to $\tilde{f}$ is a pair $(\Phi, \psi)$ satisfying (I)(a) and (b) in (2.1) Definition and

(c) $g(x, s)=\lambda\left(\Phi^{*} \tilde{f}(x, s), s\right)$ for some $\lambda$ in $\mathscr{O}_{1+l}$ with $\lambda(y, 0)=y$.

(II) A strict right morphism from $g$ to $\tilde{f}$ is a right-left morphism such that $\lambda(y, s)=y$ in $(c)$.

The following is a direct consequence of (1.2) Theorem.

(2.4) Proposition. Let $\tilde{f}$ and $g$ be unfoldings of $f$. A pair $(\Phi, \psi)$ is, respectively, a right-left morphism or a strict right morphism from $g$ to $\tilde{f}$ if and only if it is an $R L$-morphism or a morphism from $\mathscr{F}^{\prime}=(d g)$ to $\mathscr{F}=(d \tilde{f})$.

\section{REFERENCES}

1. J.-F. Mattei et R. Moussu, Holonomie et intégrales premières, Ann. Sci. Ecole Norm. Sup. 13 (1980), 469-523.

2. R. Moussu, Sur l'existence d'intégrales premières pour un germe de forme de Pfaff, Ann. Inst. Fourier (Grenoble) 26 (1976), 171-220.

3. R. Moussu et J.-C. Tougeron, Fonctions composées analytiques et defférentiables, C. R. Acad. Sci. Paris 282 (1976), 1237-1240.

4. T. Suwa, $A$ theorem of versality for unfoldings of complex analytic foliation singularities, Invent. Math. 65 (1981), 29-48.

5. __ Determinacy of analytic foliation germs, Advanced Studies in Pure Math., vol. 5, Foliations (I. Tamura, ed.), Kinokuniya and North-Holland, 1985, pp. 427-460.

6. - The versality theorem for RL-morphisms of foliation unfoldings, Advanced Studies in Pure Math., vol. 8, Complex Analytic Singularities (T. Suwa and P. Wagreich, eds.), Kinokuniya and North-Holland, 1986, pp. 599-631.

7. G. Wassermann, Stability of unfoldings, Lecture Notes in Math., vol. 393, Springer-Verlag, Berlin and New York, 1974.

Department of Mathematics, Hokkaido UniVERsity, SAPPoro, JAPAN 